\title{
VARIABILITY OF JUPITER'S SYNCHROTRON EMISSION IN MID-2009
}

\author{
D. Santos-Costa*, R. Sault ${ }^{\dagger}$, S. Bolton* ${ }^{*}$ R. Thorne ${ }^{\ddagger}$, and S. Levin ${ }^{\S}$
}

\begin{abstract}
In the present paper, radio observations made at the wavelength of $6 \mathrm{~cm}$ with the VLA in 2009 are analyzed. Reconstructed images of the brightness distribution show significant intensity variations of the equatorial peak emissions on both sides of the planet. The fluctuations are characterized by asymmetrical changes in the brightness distributions. The contribution of a comet-like impact to the observed variations is discussed. During the third week of July, ground-based measurements at different radio bands confirmed that a large projectile had struck Jupiter's atmosphere. The examination of the 2009 VLA data sets shows that the steep enhancement of the emission radiated by Jupiter's electron belt occurred during the same period. The increase in the synchrotron radiation was observed to go on for a couple of weeks before gradually fading in August. Two-dimensional reconstructions of the equatorial brightness distribution demonstrate that the time variability of the radio emission during the middle of 2009 was driven by the longitudinal expansion of an impact-related synchrotron "hot spot" originally located at the Jupiter System III longitude of 305 degrees.
\end{abstract}

\section{Introduction}

Jupiter's strong magnetic field contributes to populate the innermost region of the giant planet's magnetosphere with very energetic electrons, resulting in an observed radio signal above the galactic noise level [Drake and Hvatum, 1959]. The fast gyration of highly relativistic electrons around field lines is the basic mechanism responsible for the production of Jupiter's intense synchrotron radiation [Field, 1959]. Measured in the decimetric band since the late 1950's [McClain and Sloanaker, 1959; Roberts and Stanley, 1959; and others], this radiation led to the discovery of the electron belt in the 1960's [Carr et al., 1983]. With the little amount of existing particle data for the region inside Io's orbit,

\footnotetext{
* Southwest Research Institute, San Antonio, TX 78238-5166, USA

$\dagger$ University of Melbourne, Australia

¥Department of Atmospheric and Oceanic Sciences, UCLA, Los Angeles, CA, USA

§ Jet Propulsion Laboratory, California Institute of Technology, Pasadena, CA 91109, USA
} 
the repeatedly observation of Jupiter's synchrotron emission has been a valuable tool to provide information on the features of the radiation belt, constrain models of the energy and spatial distributions of the trapped particles and continues today to be the only way to probe the temporal variability of the Jovian relativistic electrons.

In the present paper, analysis results of observations made with the VLA in mid-2009 are presented. In the following sections, the campaign of observations and data reduction are first described before discussing our imaging results. Evidence for short-term changes in Jupiter's synchrotron emission at 6-cm wavelength is examined and the contribution of a comet-like impact to observed variations investigated.

\section{Observation and Data Reduction}

In 2009, we have observed Jupiter at 6 and 20 centimeters with the Very Large Array. Eleven observational times were scheduled between June $22^{\text {nd }}$ and September $12^{\text {th }}$. For three of these days, the emission in the decimetric wavelength was measured from Jupiter's rise to set. The measurements were limited up to 4 hours for each of the eight other days. All the observations were made with the VLA in its C-configuration [see NRAO Green Book, 1992]. The Earth's declination $\left(D_{E}\right)$ as seen from Jupiter barely fluctuated and was close to 0 degree jovigraphic. Jupiter was approximately $4.13 \mathrm{AU}$ away from Earth with an apparent angular size of 47.7". Observational times, Central Meridian Longitude (CML) coverages and information on geometric parameters are summarized in Table 1.

Table 1: Log of the VLA 2009 campaign

\begin{tabular}{ccccc}
\hline $\begin{array}{c}\text { UT date }(2009) \\
\text { month:d/hr:mn-d/hr:mn }\end{array}$ & $\begin{array}{c}\text { CML range } \\
(0)\end{array}$ & $\begin{array}{c}D_{E} \\
\left({ }^{0}\right)\end{array}$ & $\begin{array}{c}\text { Geocentric } \\
\text { distance }(A U)\end{array}$ & $\begin{array}{c}\text { Jupiter angular } \\
\text { size }(")\end{array}$ \\
\hline June 22/14:45-22/15:56 & {$[150 ; 194]$} & 0.47 & 4.41 & 44.7 \\
July 07/05:40-07/14:33 & {$[282 ; 243]$} & 0.51 & 4.23 & 46.6 \\
July 08/09:06-08/12:59 & {$[197 ; 337]$} & 0.51 & 4.23 & 46.6 \\
July 22/06:41-22/10:35 & {$[059 ; 199]$} & 0.52 & 4.11 & 48.0 \\
July 24/04:03-24/08:01 & {$[265 ; 048]$} & 0.52 & 4.10 & 48.1 \\
Aug. 03/06:23-03/10:18 & {$[056 ; 197]$} & 0.50 & 4.05 & 48.7 \\
Aug. 04/03:49-04/12:43 & {$[114 ; 076]$} & 0.50 & 4.05 & 48.7 \\
Aug. 23/07:04-23/10:59 & {$[214 ; 355]$} & 0.44 & 4.04 & 48.8 \\
Aug. 30/02:07-30/11:01 & {$[009 ; 331]$} & 0.42 & 4.06 & 48.5 \\
Aug. 31/03:33-31/07:31 & {$[212 ; 355]$} & 0.42 & 4.07 & 48.4 \\
Sept. 12/03:16-12/07:13 & {$[209 ; 351]$} & 0.37 & 4.14 & 47.6 \\
\hline
\end{tabular}

The all four Stokes parameters (I, Q, U and V) were measured during the campaign of observations but to date, only the parameter I (total intensity) has been processed and analyzed. The UV data were flagged with NRAO AIPS (Astronomical Image Processing Software) then reduced with the MIRIAD (Multichannel Image Reconstruction Image Analysis and Display) package software [Sault et al., 1995]. After being calibrated, the visibility data were processed with the MIRIAD tasks INVERT, MAXEN and CONVOL to produce maps of brightness distribution. The thermal emission was subtracted during 
the data reduction to provide only images of Jupiter's synchrotron emission. As discussed by Santos-Costa et al. [2009], we can concede a $5 \%$ error on the data values of the "Clean" images.

\section{Brightness Distribution at $6 \mathrm{~cm}$}

\subsection{Two-dimensional maps}

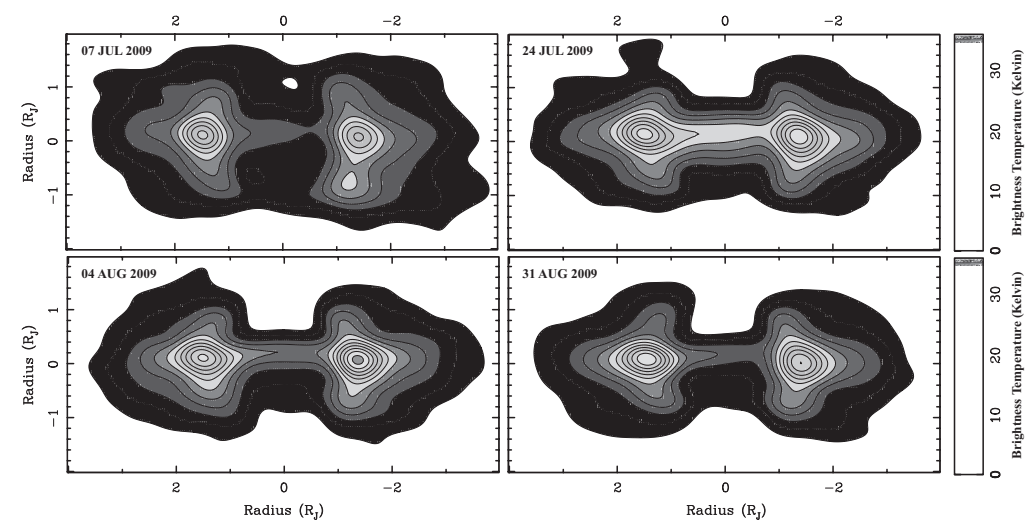

Figure 1: Maps of Jupiter's synchrotron emission at the wavelength of $6 \mathrm{~cm}$ and CML of $340^{\circ}$. Maps display significant changes in the brightness distribution on a scale of weeks.

Total intensity maps of Jupiter's synchrotron emission were built from measurements made with the VLA in 2009. Nine images were constructed every $40^{\circ}$ of Central Meridian Longitude. For each CML, data within $\pm 20^{\circ}$ of that CML were combined. During the data reduction, the effects of the magnetic field wobble were corrected and the flux density was converted to brightness temperature $T_{B}$. Images were degraded to the same image resolution to permit comparisons between different observational days. Figure 1 displays two-dimensional maps of the synchrotron radiation in total intensity for the wavelength of $6 \mathrm{~cm}$ and $\mathrm{CML}$ of $340^{\circ}$. Each map has the magnetic axis vertical and the displayed data have values between 0 and $36 K$.

Figure 1 shows noticeable changes in the brightness distribution of Jupiter decimetric emission. On 07 Jul 2009, the brightness peak, defined as the brightest peak emission, was located on the left-hand (east) side of Jupiter (left top panel). Seventeen days later, on 24 Jul 2009, brightness temperatures were observed to have increased on both sides of the planet (right top panel). The east peak emission increased by about $12 \%$ while the west one did by $37 \%$. Emission radiated by the electrons drifting in front of the planet was also stronger, brighter by a factor two. Due to the non-symmetrical enhancement of the peak emissions, the brightness peak shifted to the west side of the planet. Emission from the right-hand side of the planet was still the most intense on 04 Aug 2009 (left bottom 
panel). On 31 Aug 2009, the east lobe became again the brighter lobe after the west peak emission sensitively decreased by about ten percentage points. Figure 1 presents the first evidence for short-time variations observed during the middle of 2009.

\subsection{East-West Asymmetry}
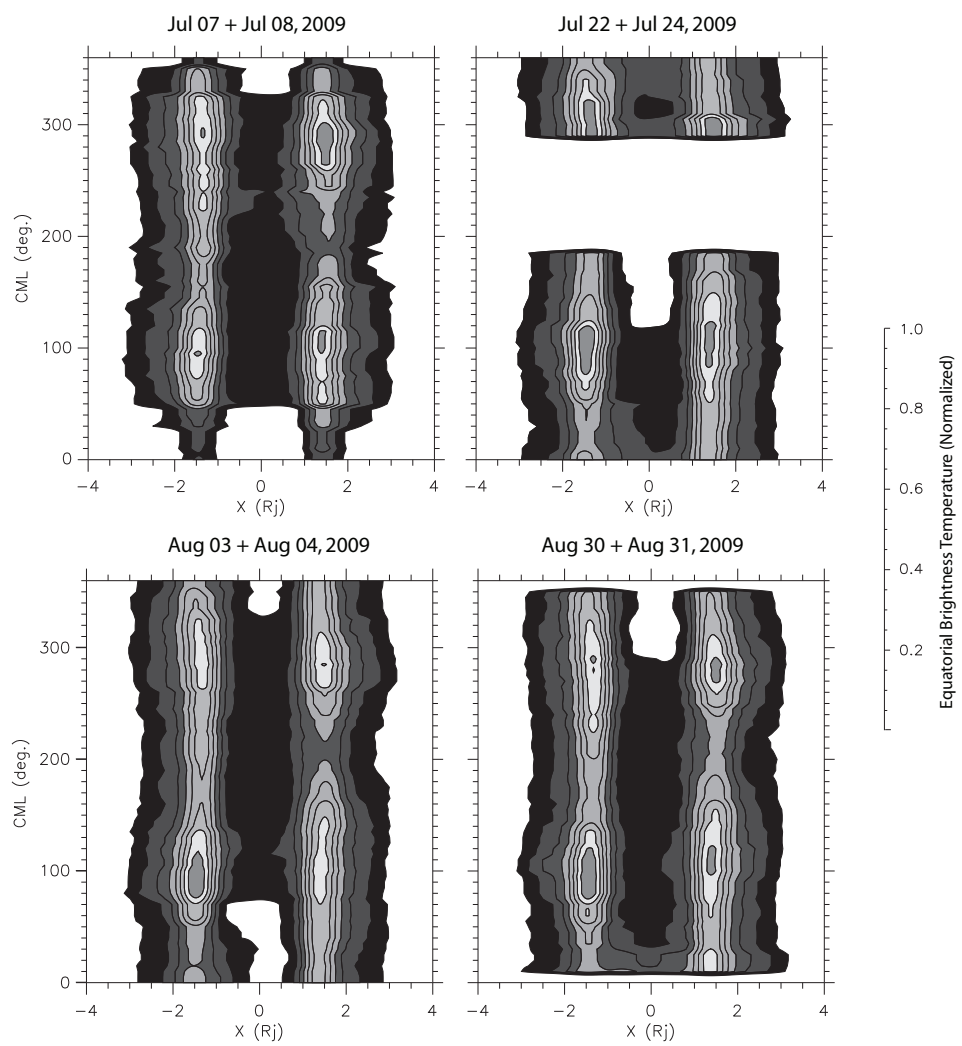

Figure 2: $C M L$-versus-distance spectrograms of the equatorial brightness temperature at $6 \mathrm{~cm}$.

To perform a detailed examination of the changes in the Jovian synchrotron emission's east-west brightness distribution as a function of CML, new images were built every five degrees with a data coverage of $20^{\circ}\left(\mathrm{CML} \pm 10^{\circ}\right)$ instead of $40^{\circ}$. To reduce the amount of data to process and analyze, observations from two consecutive observational days were merged together. Measurements made on July $7^{\text {th }}$ (respectively July $22^{\text {nd }}$, Aug. $3^{\text {rd }}$, and Aug. $30^{\text {th }}$ ) were combined with those of July $8^{\text {th }}$ (respectively July $24^{\text {th }}$, Aug. $4^{\text {th }}$, and Aug. $31^{\text {st }}$ ). Merging data sets by pair of observational days limits our study of Jupiter's electron belt dynamics on a scale of weeks. Nonetheless, Figure 2 shows interesting results. 
In Figure 2, the CML-versus-distance spectrograms of the brightness temperature were obtained by taking a slice from each new map constructed at $6 \mathrm{~cm}$. Slices of $0.4-\mathrm{R}_{J}$ thickness were taken through the Jovian magnetic equator and stacked together. For each panel, the displayed data values were normalized to the highest temperature. Figure 2 demonstrates that the rocking of the brightness peak from one side of the planet to the other occurred at other longitudes than $340^{\circ}$. This was mainly noticed at CMLs in the $\left[70^{\circ} ; 130^{\circ}\right]$ and $\left[260^{\circ} ; 360^{\circ}\right]$ angular sectors. The observed shift at a given CML was controlled by fluctuations of the equatorial peak emission occurring on either one or both sides. The intensity of each lobe was varying between 10 and $40 \%$. For the other longitudes, the brightness peak remained located on the same side of the planet. Only significant fluctuations on both sides of Jupiter were remarkable, with variations as important as 30 up to $35 \%$.

The unmistakable temporal variations of the equatorial brightness temperature observed in mid-2009 but the likeness between those displayed on the upper left and lower right panels of Figure 2 indicate that the electron radiation-belt populations were undergoing significant changes in their energy and spatial distributions during a period that lasted between 4 and 6 weeks (likely from mid-July to early September).

\section{Response to a Comet-like Impact}

During the third week of July 2009, ground-based measurements at different radio bands confirmed that a large projectile had struck Jupiter's atmosphere. First noticed on July 19, 2009 in visible light as a new "black scar" near Jupiter's south pole, additional images corroborated that what was observed in near infrared as a "bright spot" was not just a weather-related disturbance but was an impact of an object. The impact site was located at Jupiter's System III longitude $\sim 305^{\circ}$ and a latitude of about $-55^{\circ}$ [Sánchez-Lavega et al., 2010].

During the collision of Comet Shoemaker-Levy 9 into Jupiter's atmosphere in 1994, shorttime changes in the brightness distribution of Jupiter's synchrotron emission were observed right after the first comet fragments started striking Jupiter. Rapid fluctuations were measured and the continuous monitoring of the signal before, during and after the 1994 event showed that the intensity variations associated with the impacts went on for a couple of weeks [Harrington et al., 2004; therein and others].

To discuss the contribution of the July 2009 impact to the intensity variations observed in mid-2009, we examine reconstructed maps of equatorial brightness distribution. The latter were built by solving a system of linear equations, $A_{m \times n} \cdot X_{n}=B_{m}$, using the SVD method [Lawson and Hanson, 1995]. Index $\mathrm{m}$ is defined by $N_{C M L} \times N_{p x}$ with $N_{C M L}$ $(\leq 72)$ the number of brightness distribution maps (as displayed in Figure 1) available for each set of observational days and $N_{p x}(=196)$ the number of pixels providing a value for the brightness temperature $T_{p x}(R)$ along the magnetic equator $(-4 \leq R \leq+4$ $R_{J}$ ). To take into account the effects of the magnetic field wobble and ensure that the small variation of $D_{E}$ was not biasing our calculation of brightness temperatures, $T_{p x}$ was averaged over a few vertical pixels. This was equivalent to take slices of $0.4-R_{J}$ thickness 

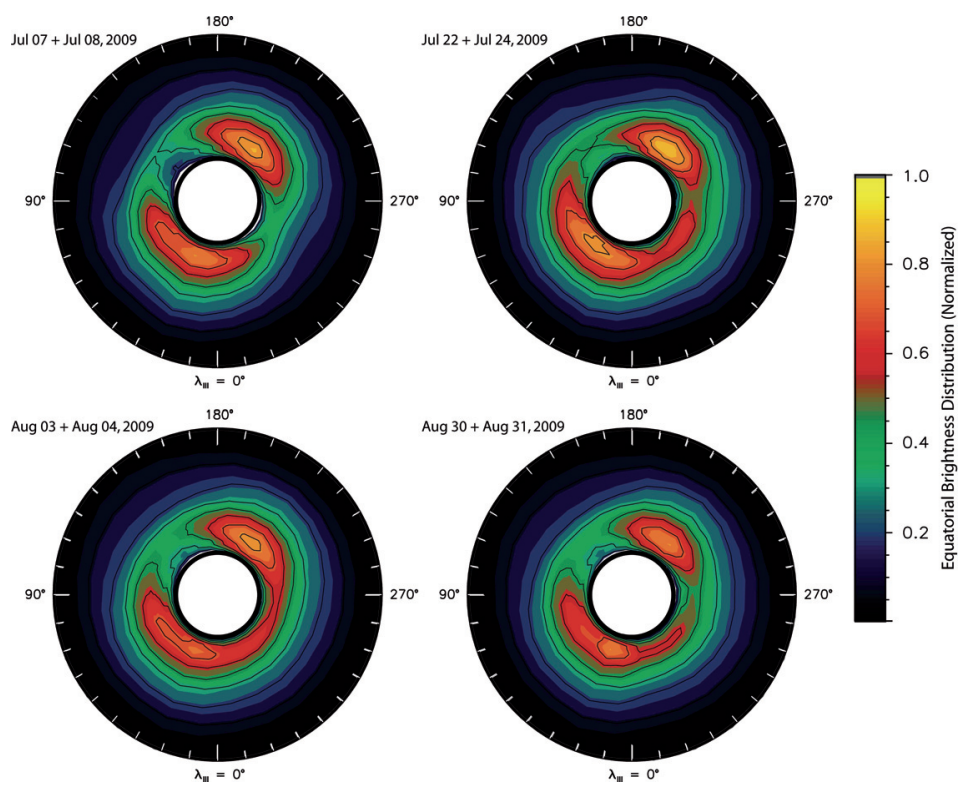

Figure 3: Maps of equatorial brightness distribution at $6 \mathrm{~cm}$ displaying the longitudinal dispersion of a new synchrotron "hot spot" first located at $\lambda_{I I I} \sim 305^{\circ}$ during the middle of 2009.

along the equator and assume that near equatorial electrons have "pancake" pitch-angle distributions. Index $n=n_{r} \times n_{\lambda_{I I I}}=40 \times 36$ is our hypothetical number of equatorial point sources $S\left(r, \lambda_{I I I}\right)$ of synchrotron radiation $\left(1 \leq r \leq 4 \mathrm{R}_{J}\right.$ and $\left.0 \leq \lambda_{I I I} \leq 360^{\circ}\right)$. For each pixel at a given CML and for a window of observation, the expression of the brightness temperature is [Santos-Costa, 2001]

$$
T_{p x}(C M L, R)=\frac{\lambda_{o b s}^{2} R_{J}}{2 k} \int_{-4}^{+4} \varepsilon\left(c / \lambda_{o b s}, l\right) d l=\zeta\left(\lambda_{o b s}\right) \int_{-4}^{+4} \varepsilon d l,
$$

where $\varepsilon\left(c / \lambda_{o b s}, l\right)$ is the volume emissivity (power per unit frequency interval per unit volume per unit solid angle) of an equatorial source of radiation-belt emission located along the line of sight. $\lambda_{o b s}$ is the wavelength of observation and the constants $k$ and $c$ are the Boltzmann constant and speed of light, respectively. In $A . X=B$, the matrix $X$ thus contents the $n$ volume emissivities $\varepsilon_{n_{r} \times n_{\lambda_{I I I}}}$ to be computed and $B$ contents the $N_{C M L} \times N_{p x}$ brightness temperatures. The coefficient matrix $A$ satisfies $B_{i}=\zeta\left(\lambda_{o b s}\right) \sum_{j=1}^{n} A_{i, j} \varepsilon_{j}(i=$ $1, m)$ and must be determined before solving the linear system.

Figure 3 displays the result of our numerical resolution of the linear system $A_{m \times n} . X_{n}=B_{m}$ for four windows of observation. The calculation of the equatorial brightness distributions had required the resolution of linear systems with 10,000 up to 15,000 equations. For each of the four maps of Figure 3, the temperatures $T_{B}$ were normalized to the highest temperature. All four distributions show the presence of two 70-degree sectors of intense 
radiation at $r \sim 1.4 \mathrm{R}_{J}$. The non-axisymmetric confinement of near equatorial energetic electrons, resulting from the configuration of both the planetary magnetic field inside about $5 \mathrm{R}_{J}$ (equivalent to an offset and tilted dipole) and ring system, is responsible for the asymmetrical distribution of the equatorial brightness temperature.

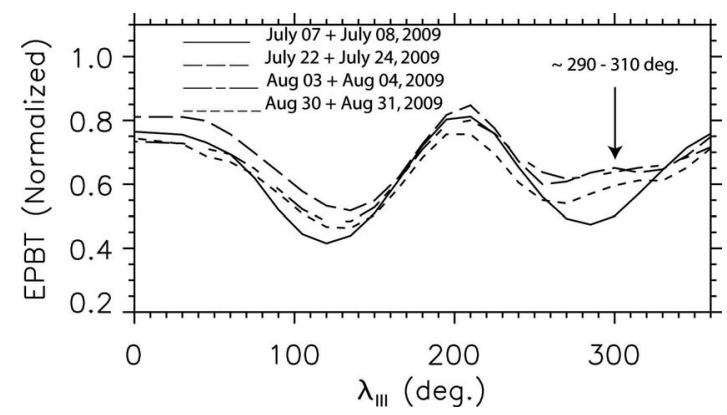

\begin{abstract}
Figure 4: $\quad$ Longitude profiles of the equatorial peak brightness temperature reconstructed from equatorial distributions. The formation then dispersion of a synchrotron "hot spot" first located at $\lambda_{I I I} \sim 290-310^{\circ}$ after the July 2009 impact are confirmed.
\end{abstract}

What is also important to note in Figure 3 is the presence of a new synchrotron "hot spot" at $\lambda_{I I I} \sim 290-310^{\circ}$ that apparently appeared sometime after mid-July (top right map) and had longitudinally diffused during the following five weeks (bottom two maps). The equatorial peak brightness temperature profiles as a function of longitude reconstructed from the equatorial brightness distributions computed at $6 \mathrm{~cm}$ confirm this result (Figure 4). The increase of thirty percentage points in the peak brightness temperature at $\lambda_{I I I} \approx$ $305^{\circ}$ is responsible for the apparition of a temporary "hot spot" in Jupiter's electron belt. The longitudinal dispersion and change in intensity of this "hot spot" between mid-July and early September certainly explain the asymmetrical intensity variations of Jupiter's synchrotron emission observed at $6 \mathrm{~cm}$ during the middle of 2009 (Figures 1 and 2).

\title{
5 Conclusion
}

In the present paper, radio measurements collected in 2009 with the Very Large Array were examined. The observations were made at the wavelengths of $6 \mathrm{~cm}$ and $20 \mathrm{~cm}$ but only data analysis results for the shorter wavelength were presented. With 11 observational days spread out over a period of 83 days, the dynamical behavior of Jupiter's synchrotron emission on scales of days to weeks was investigated. The investigation was made possible by first examining brightness distribution maps of the total intensity and one-dimensional scans of the equatorial brightness temperature.

Brightness distribution maps at $6 \mathrm{~cm}$-wavelength demonstrate that the spatial structure of Jupiter's radiation-belt emission was changing on short time-scales, thus confirming the capability of observing rapid variations of the non-thermal emission of this planet with interferometers [Santos-Costa et al., 2009]. In 2009, the brightness temperatures along the magnetic equator were again observed to evolve differently during Jupiter's 10-hour rotation and over a long period of observations. The peak emissions on both sides of the Jovian planet were significantly fluctuating. At times, these changes were characterized by a shift of the brightness peak from one side of the planet to the other. 
To determine the origin of the fluctuations observed in mid-2009, two-dimensional maps of the equatorial brightness distribution were build. The tomographic images were reconstructed from the brightness temperatures measured along the magnetic equator at different longitudes and windows of observations. They have required the resolution of systems of linear equations using the SVD method. Longitude profiles of the equatorial peak brightness temperature were then reconstructed and analyzed.

Maps and profiles of the equatorial brightness temperature at 6-cm wavelength demonstrate that a synchrotron "hot spot" in Jupiter's electron belt appeared right after the July 2009 impact. An increase of thirty percent points in the brightness temperature at $\lambda_{I I I} \sim 290-310^{\circ}$ was responsible for its formation. The longitudinal dispersion and change in intensity of this new "hot spot" during a 5-6 week period are believed to explain the asymmetrical intensity variations of Jupiter's synchrotron emission in mid-2009.

Due to similarities with the variability of Jupiter's synchrotron emission after the collision of Comet Shoemaker-Levy 9 into Jupiter in 1994, Santos-Costa et al. ['Variability of Jupiter's radiation-belt emission during the middle of 2009: Observation and interpretation', J. Geophys. Res., in revision] have further investigated the response of this radiation to the July 2009 event by reconstructing the time profile of the total flux from the 2009 VLA observations and discuss the mechanisms at play after the impact.

\section{References}

Carr, T. D., M. D. Desch, and J. K. Alexander, Phenomenology of magnetospheric radio emission, In Physics of the Jovian magnetosphere, edited by A. J. Dessler, Cambridge University Press, 226-284, 1983.

Drake, F. D., and H. Hvatum, Non-thermal microwave radiation from Jupiter, Astron. J., 64, 329-330, 1959.

Field, G. B., The source of radiation from Jupiter at decimeter wavelengths, J. Geophys. Res., 64, 1169-1177, 1959.

Harrington, J., I. de Pater, S.H. Brecht, D. Deming, V. Meadows, K. Zahnle, and P. D. Nicholson, Lessons from Shoemaker-Levy 9 about Jupiter and planetary impacts, In Jupiter. The planet, satellites and magnetosphere, edited by F. Bagenal, T. E. Dowling, and W. B. McKinnon, Cambridge University Press, 1, 159-184, 2004.

Lawson, CL., and R. J. Hanson, Solving least squares problems, Classics in Applied Mathematics series, SIAM edition, 1995.

McClain, E. F., and R. M. Sloanaker, Preliminary observations at $10 \mathrm{~cm}$ wavelength using the NRL 84-foot radio telescope, in Proceedings IAU Symposium No. 9 URSI Symposium No. 1, edited by R. Bracewell, Stanford University Press, 61-68, 1959.

NRAO Green Book, An introduction of the NRAO Very Large Array, edited by R. M. Hjellming, NRAO Publ., 1992. 
Roberts, J.A., and G. J. Stanley, Radio emission from Jupiter at a wavelength of 31 centimeters, Publ. Astron. Soc. Pacific., 71, 485-496, 1959.

Sánchez-Lavega, A., A. Wesley, G. Orton, R. Hueso, S. Perez-Hoyos, L. N. Fletcher, P. Yanamandra-Fisher, J. Legarreta, I. de Pater, H. Hammel, A. Simon-Miller, J. M. Gomez-Forrellad, J. L. Ortiz, E. García-Melendo, R. C. Puetter, and P. Chodas, The impact of a large object on Jupiter in 2009 July, Astrophys. J. Lett., 715, 2, L155-L159, 2010.

Santos-Costa, D., Modélisation des ceintures de radiation d'électrons de Jupiter internes à Io, ENSAE thesis, 2001.

Santos-Costa, D., S. J. Bolton, and R. J. Sault, Evidence for short-term variability of Jupiter's decimetric emission from VLA observations, Astron. Astrophys., 508, 2, 1001-1010, 2009.

Sault, R. J., P. J. Teuben, and M. C.H. Wright, A retrospective view of MIRIAD, Astronomical Data Analysis Software and Systems IV, ASP Conference Series, 77, 433, 1995 . 
